\title{
Investigating projectile penetration of concrete blocks reinforced by ceramics
}

\author{
R. Tawadrous ${ }^{1}$, W. Attia ${ }^{1} \&$ M. Laissy ${ }^{2}$ \\ ${ }^{1}$ Structural Engineering Department, Faculty of Engineering, \\ Cairo University, Egypt \\ ${ }^{2}$ Faculty of Engineering, MTI University, Egypt
}

\begin{abstract}
Numerical simulation of the response of concrete structures to impact loading is an important tool in both the design of hardened protective structures and in the planning for effective attacks against such structures. This paper presents the development of an accurate finite-element model using AUTODYN to study the response of concrete structures reinforced by ceramic (Al2O3-99.7\%) rods and plates exposed to $23 \mathrm{~mm}$ projectile. Concrete and ceramic are modelled using a combined mesh and meshfree numerical technique. The used meshfree Lagrangian technique (SPH) is to overcome problems of mesh tangling and remove the requirement for the use of erosion algorithms. The technique also allows an explicit representation of Ceramic through (SPH) element formulation. In such a model, the concrete region local to the penetrator, which experiences large deformation, is represented using the SPH solver. The modelled penetrator and the concrete further away from the impact observed to undergo little or no deformation by using the Lagrange solver.

The aim of this paper is to study numerically the penetration resistance of concrete structures reinforced by ceramic (Al2O3-99.7\%). The main findings show an enhancement in the penetration resistance of about $45 \%$ while using ceramic. Here, we used ceramic because of its electric, magnetic, and thermal insulation. Hence, we can use concrete structures reinforced by ceramic in many types of medical, nuclear, power generating and electronic applications.

Keywords: penetration, concrete, ballistic impact, ceramic, AUTODYN3D.
\end{abstract}




\section{Introduction}

In civilian and military applications, over the years concrete is used as a construction material for construction of protective structures. Great demand exists for designing of nuclear plants, power plants, military structures, water retaining structures, highway barriers, etc., to resist the penetration and perforation of concrete structure against kinetic projectile, generated both accidentally and deliberately, in various impact and blast scenarios. (e.g. failure of a pressurized vessel, failure of a turbine blade or other high speed rotating machines, aircraft crashes, fragments generated by accidental explosions, etc.); terrorist attack, and natural disasters like tsunami and etc. Critical impact energy is the dominant cause of damage in dynamic of local impact phenomena $[1,2]$. When hard projectile impacts with concrete target, critical impact energy of the projectile is a main reason that makes the concrete target deform. Therefore, critical impact energy, which can cause penetration and perforation in concrete structures, is also noteworthy in determining the dynamic response of concrete structures against the penetration and perforation of hard projectile [1, 2]. In this paper, a numerical simulation study is conducted to show the effect of using ceramic (A12O3-99.7\%) rods and plates as a reinforcement to concrete target.

There are three groups of models. First group the target is plain concrete to validate the numerical simulation according to experimental investigation that was performed by Mohamed et al. [3]. In which steel blunt-nose projectile with a diameter of $23 \mathrm{~mm}$ and a mass of 175 grams is fired with striking velocity about $960 \mathrm{~m} / \mathrm{s}$ to hit the target, and the penetration depth was $400 \mathrm{~mm}$. In the second group, the target is concrete reinforced by ceramic (A12O3-99.7\%) rods. The third group is concrete reinforced by (A12O3-99.7\%) ceramic plate as a sandwich panel.

\section{Background}

Local and overall impact phenomena; for hard missile impact are schematically shown in figure 1 . With very low velocities, the missile will strike the target wall and bounce off without creating any local damage. As the velocity increases, pieces of concrete are spalled (ejected) off the front of impacted face of the target. This spalling forms a spall crater that extends over a substantially bigger area than the cross-sectional area of the striking projectile. As the velocity continues to increase, the projectile will penetrate the target to depths beyond the depth of the spall crater, forming a cylindrical penetration hole with a diameter only slightly bigger than the missile diameter. As the penetration depth increases, the projectile will stick to the concrete target rather than rebounding. At this stage, the impact meets the criteria of a plastic impact. However, even at lesser penetration depths, we can treat the impact approximately as a plastic impact when determining the energy absorbed by the impacted target. Further increase in velocity produces cracking of the concrete on the back surface followed by scabbing (ejection) of concrete from this rear surface. The zone of scabbing will generally be much wider but not as deep as the front-face crater. 
Once scabbing begins, the depth of penetration will increase rapidly. For low barrier thickness-to-projectile, diameter ratio $(<5)$ the pieces of scabbed concrete can be large and have substantial velocities. As the projectile velocity increases further, perforation of the target will occur as the penetration hole extends through to the scabbing crater. Still higher velocities will cause the projectile to exit from the rear face of the target. Upon plastic impact, portions of the total kinetic energy of the impacting projectile converted to strain energy associated with deformability of the projectile and energy losses associated with target penetration. The reminder of the energy is absorbed or given as input to the target. This absorbed energy results in overall target response that includes flexural deformation of the target barrier and deformation of its supporting structure.

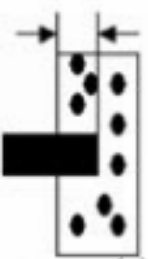

(a)

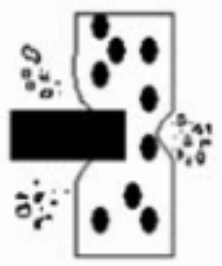

(e)

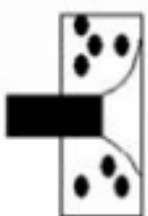

(b)

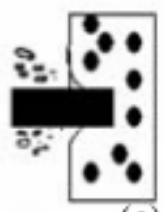

(c)

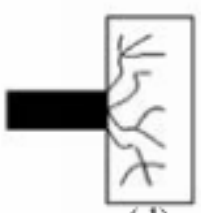

(d)

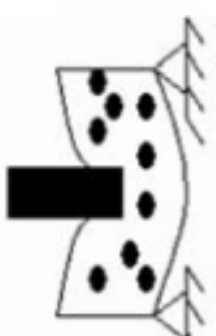

(g)

Figure 1: Missile impact effects on concrete target: (a) penetration, (b) cone cracking, (c) spalling, (d) cracking, (e) scabbing, (f) perforation, and (g) overall target response.

\section{Numerical analysis}

The efficient and accurate numerical prediction of kinetic energy penetrator impacts on the concrete structure requires three basic components; appropriate numerical techniques, a set of constitutive laws and material data input to the constitutive laws. Here a description of a combined mesh and meshfree approach developed in the AUTODYN software [4] and used for the simulation of projectile impacts onto plain and reinforced concrete.

The concrete target represented numerically by a mesh based Lagrangian technique except in the regions where high deformations are expected. Here, a meshfree Lagrangian technique (SPH) used to overcome problems of mesh 
tangling and remove the requirement for the use of erosion algorithms. Technique for representing continuous joins between mesh and meshfree Lagrangian techniques presented [5]. Ceramic represented explicitly through a meshfree Lagrangian technique (SPH) element formulation. The concrete region local to the penetrator, which experiences large deformation, represented using the SPH solver. The modelled penetrator and the concrete further away from the impact observed to undergo little or no deformation by using the Lagrange solver.

\subsection{Mesh based Lagrangian technique}

The Lagrange solver, in which the numerical grid distorts with the material, has the advantage of being computationally efficient and gives a good representation of material interfaces [5]. For penetration simulations, however, the grid quickly distorts in regions local to the penetrator. This gives rise to small calculation time steps and a reduction in efficiency. Eventually, cells will become tangled (degenerate) and the calculation will stop. To improve efficiency, highly distorted cells automatically removed from the calculation using an erosion algorithm based on plastic or geometric strain. The erosion process is not physically based and leads to the removal of internal material energy and if selected, kinetic energy.

\subsection{Meshfree Lagrangian technique}

Meshfree Lagrangian techniques, such as the SPH solver in AUTODYN, have the advantage of being able to deal with large deformations without the need for erosion, and at the same time are able to give a good representation of material boundaries. In AUTODYN, the complex constitutive laws developed for gridbased Lagrangian schemes can also be used directly in the SPH solver without modification.

The SPH technique is relatively new to computational continuum dynamics and is not as mature as the other grid-based techniques. Under certain conditions, numerical inconsistencies can give rise to undesirable local perturbations in the solution. To minimize these problems, it is advisable to use particles of the same size (smoothing length) within a given simulation. This requirement prevents the use of particle size transitions and therefore, leads to large model sizes if accurate simulation results are sought. This is particularly a problem in 3dimensional simulations.

\subsection{Coupling of meshfree Lagrange (SPH) to grid based Lagrange [5]}

A technique for coupling meshfree Lagrangian solvers to the Lagrange solvers has been developed and implemented in AUTODYN-2D and 3D. In this technique, the methodology for zone dimension change across the grid interface [5] has been extended to meshfree solvers. The implementation allows (as shown in figure 2), independent grids to be joined together without the interface nodes of both grids being necessarily coincident. This is particularly useful to create 
zonal transitions and effective coupling since experience [5] suggests that similar accuracy achieved when SPH particles are approximately half the size of Lagrangian cells. When nodes are coincident, we can apply this technique to couple SPH with beam elements.

For three-dimensional cases, we will define a surface connecting all SPH nodes at the interface. For this purpose, a convex hull algorithm, generally used for mesh generation, has been developed and implemented in AUTODYN-3D. As illustrated in figure 3 , we used the algorithm to triangulate the exterior envelope of an SPH object, which can thereafter be coupled to Lagrangian grids.

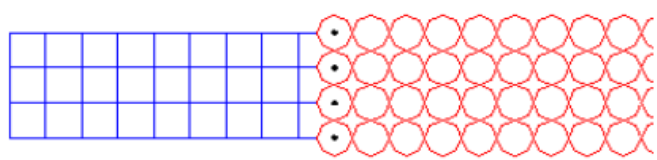

Node to Node joins

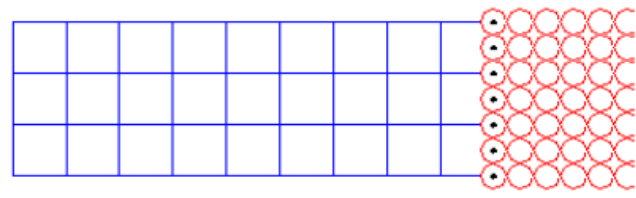

Node and Midface Joins

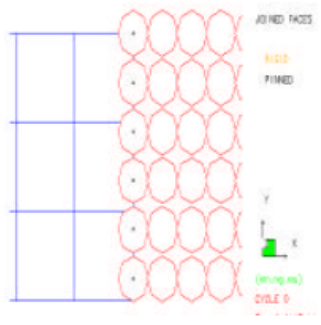

Arbitrary Node to Face joins

Figure 2: Nodal spacing for SPH to Lagrange coupling.

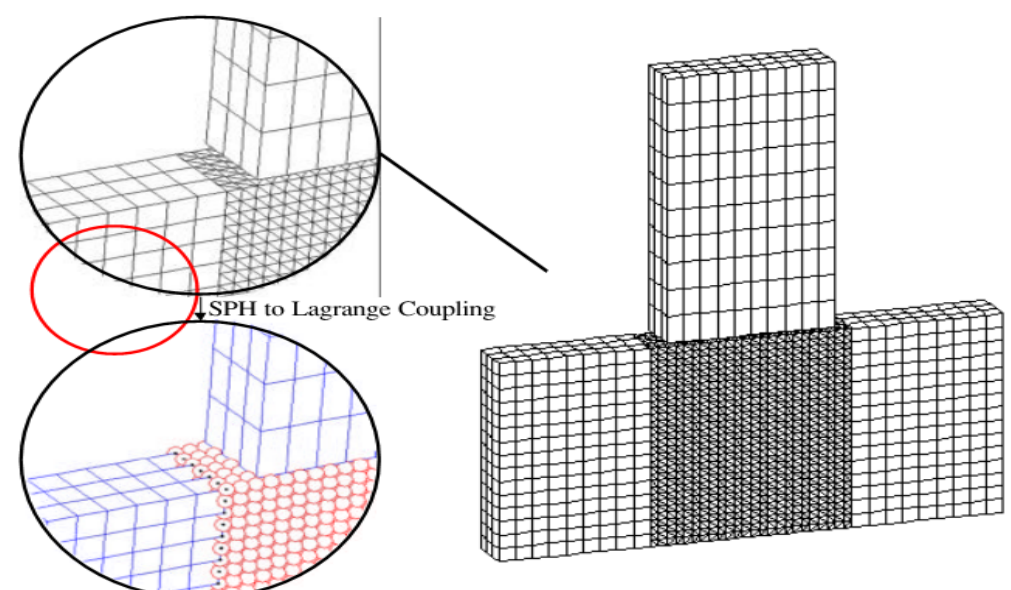

Figure 3: Triangulation of SPH external envelope using Convex Hull and coupling to Lagrange. 


\subsection{Material description}

The material model used to simulate the projectile in the model is (STEEL 4340), for plain concrete material (Conc. 35MPa) and ceramic material (AL20399.7) was used these materials were chosen from the AUTODYN library. For Steel, the equation of state used is linear equation of state, and the strength model is the Johnson Cook strength model, whereas the failure model was (None) and the selected erosion model was the Instantaneous geometrical strain.

For Concrete, the equation of state used is P-Alpha equation of state, and the strength model was RHT CONCRETE strength model with applying the modifications to the standard RHT model according to [6], whereas the failure model was RHT CONCRETE "Strength Model for the Concrete" and the erosion model was selected to be the Instantaneous geometrical strain.

The ceramic equation of state is polynomial while the strength model is JohnsonHolmquist (JH2), whereas the failure model was Johnson-Holmquist. The taken Geometrical Erosion strain is 2.0 (Instantaneous).

\subsection{Geometry and mesh description}

In AUTODYN for the analyses, we have used Lagrange and SPH solvers. In this paper, the represented Ceramic is explicitly through a meshfree Lagrangian technique (SPH) element formulation. The concrete region local to the penetrator (200 mm x $200 \mathrm{~mm}$ ), which experiences large deformation, represented using the SPH solver. The modelled penetrator and the concrete further away from the impact observed to undergo little or no deformation by using the Lagrange solver. All parts were symmetric on $\mathrm{X}=0$ and $\mathrm{Y}=0$ planes to reduce the size of the computational domain due to the symmetric conditions. The projectile geometry which is $23 \mathrm{~mm}$ diameter and $64 \mathrm{~mm}$ in length is modelled as a $1 / 4$ cylinder, it was divided to nodes in the $\mathrm{i}, \mathrm{j}$, k-directions. This ijk-index is known as a Cartesian co-ordinate system in figure 4 , which shows the geometry and mesh description for the projectile part.

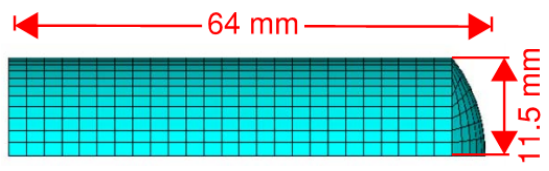

Figure 4: $23 \mathrm{~mm}$ API projectile mesh.

Group (1) the formed target model was of plain concrete as illustrated in figure 5. The model boundary condition was fixed from the side and the $23 \mathrm{~mm}$ projectile has Z-velocity initial condition of $960 \mathrm{~m} / \mathrm{s}$.

Group (2) the formed target model was of concrete reinforced by ceramic rods. Figure 6 shows the rod layers arrangement. There are two types of layers; the first one $\left(1^{\text {st }}\right.$ Type) is regular arrangement of layers through the concrete block. The second type ( $2^{\text {nd }}$ Type) of layers arrangement is staggered arrangement through the concrete block target as shown in figure 6 . 


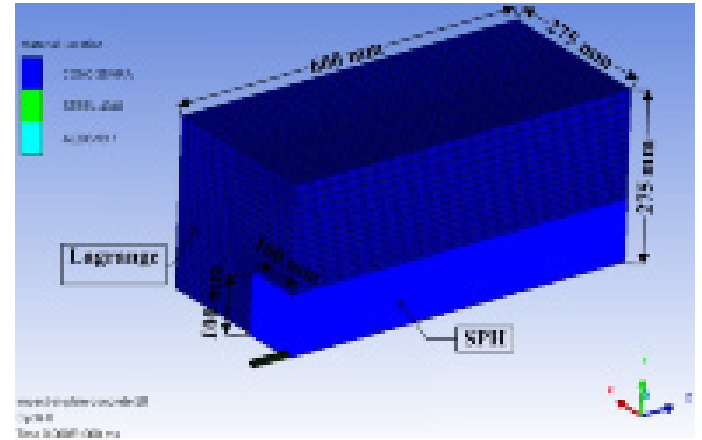

Figure 5: $\quad$ Group (1) details of model (Case 1).

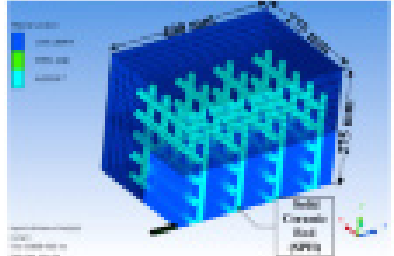

(a)

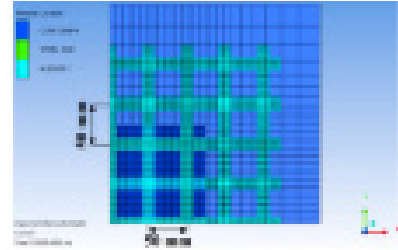

(c)

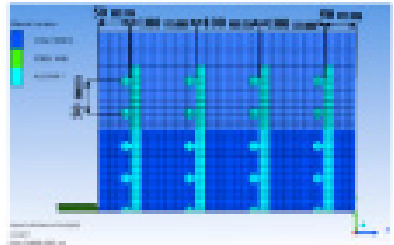

(b)

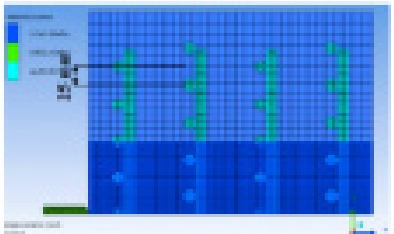

(d)

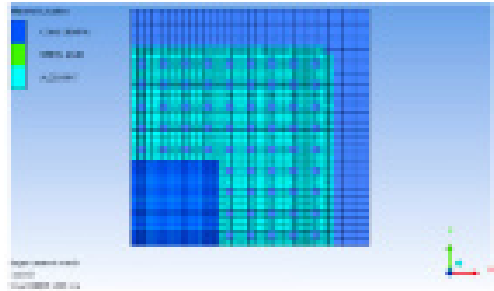

(e)

Figure 6: Geometry details of group (2): (a) 3D view; (b) Top view of regular layers arrangement; (c) Front view of regular layers arrangement; (d) Side view of staggered layers arrangement; (e) Front view of staggered layers arrangement. 
Through one layer, we distributed the ceramic rods to form a mesh of $50 \times 50 \mathrm{~mm}$ while using a $16 \mathrm{~mm}$ ceramic rod diameter. Different cases for the projectile location with respect to the rod layers are shown in figure 7.

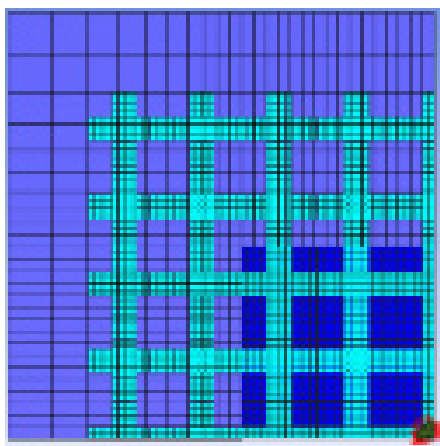

Case (2) the projectile hits two rods per layer $\left(1^{\text {st }}\right.$ Type $)$

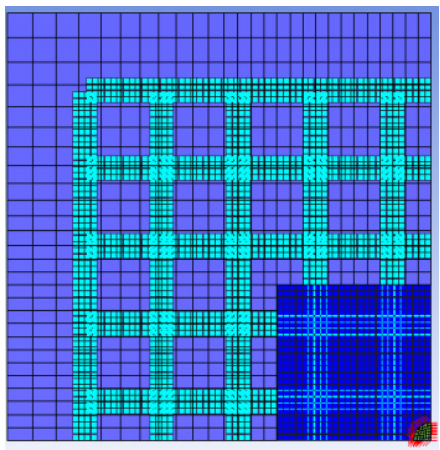

Case (4) the projectile passes through Meshes centre ( $1^{\text {st }}$ Type $)$

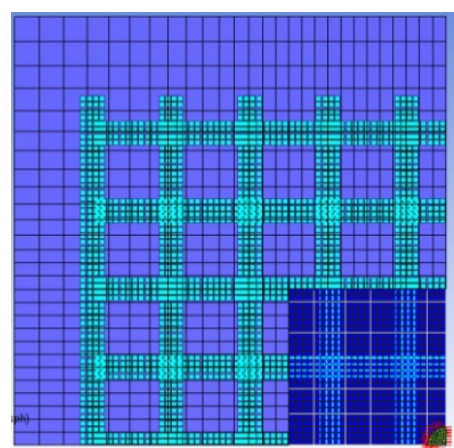

Case (3) the projectile hits one rod per layer $\left(1^{\text {st }}\right.$ Type $)$

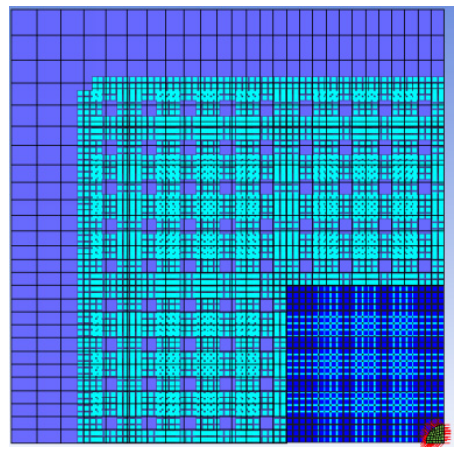

Case (5) the projectile hits two rods per layer $\left(2^{\text {nd }}\right.$ Type $)$

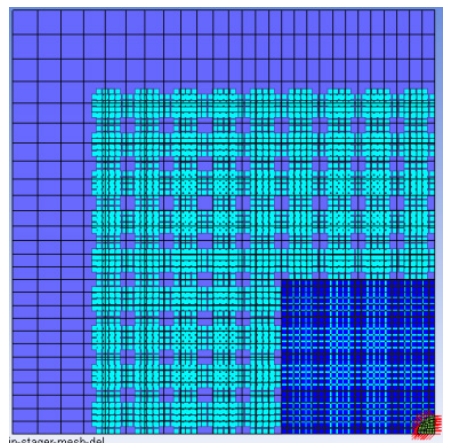

Case (6) the projectile passes through Meshes centre ( $2^{\text {nd }}$ Type $)$

Figure 7: Projectile location with respect to the rod layers. 
Group (3) formed the target model of concrete reinforced by ceramic plate as a sandwich panel as illustrated in figure 8 .

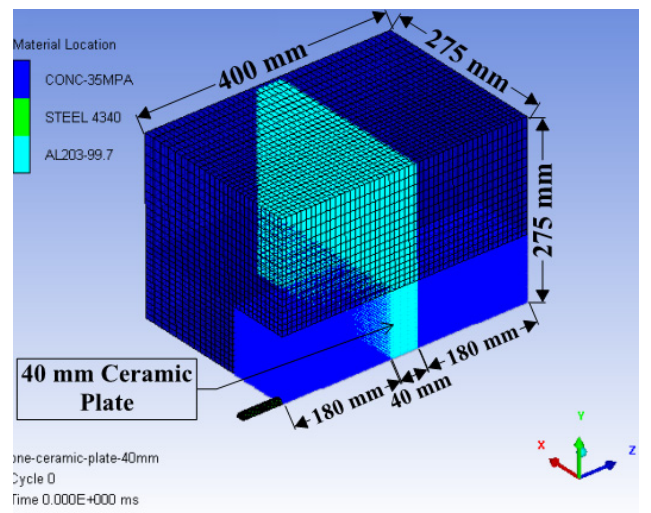

Figure 8: $\quad$ Geometry details of group (3) (Case (7)).

\subsection{Numerical test results}

Results are shown in table 1. The penetration depth for group (1) of plain concrete is $390 \mathrm{~mm}$ (Case (1)) show relatively good agreement with the experimental test by Mohamed et al. [3]. For group (2) we observed that using staggered ceramic rod layers arrangement reduce the penetration depth about $30 \%$ (case (6) the projectile passes through the weakest mesh point in staggered arrangement). While using ceramic plates as shown in-group (3) at the middle of the concrete block shows enhancement of about $45 \%$ in resultant penetration depth.

\section{Conclusion}

From the previous study, the following conclusion can be drawn out:

1. The AUTODYNE code satisfactory simulates the penetration experimental tests.

2. The response of concrete panel under the penetration load can be simulated using ANSYS software, it has the advantage, and thus it has higher analysis precision compared to the common analysis.

3. Using ceramic (A12O3-99.7\%) plates and rods as a concrete reinforcement reduced the projectile penetration depth by $45 \%$ according to this study.

4. Ceramic (Al2O3-99.7\%) plates and rods are highly recommended not only for protective structures but also for medical, nuclear, power generating and electronic applications, because of its electric, magnetic, and thermal insulation. 
122 Structures Under Shock and Impact XIII

Table 1: Numerical result of penetration resistance.

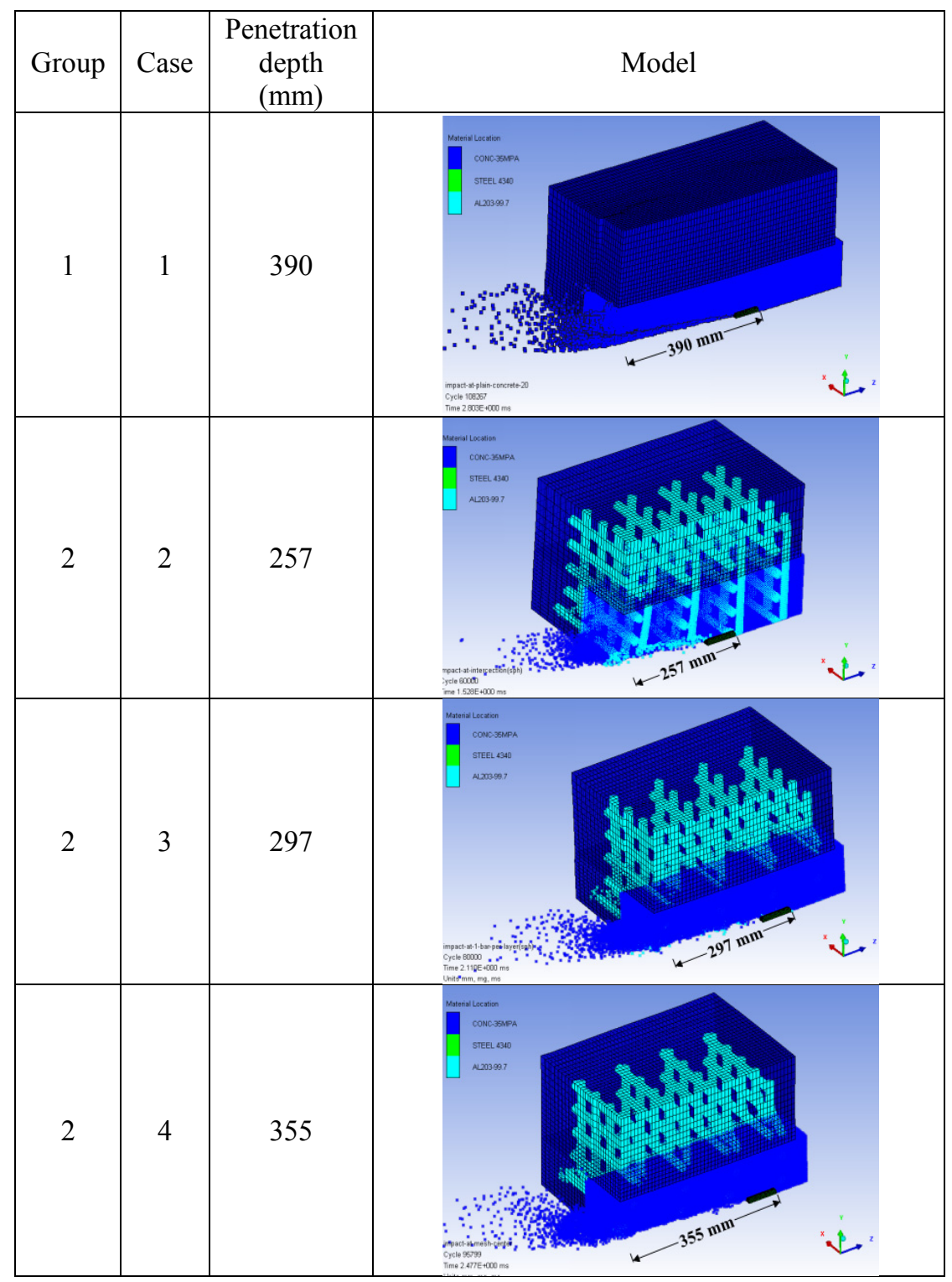


Table 1: $\quad$ Continued.

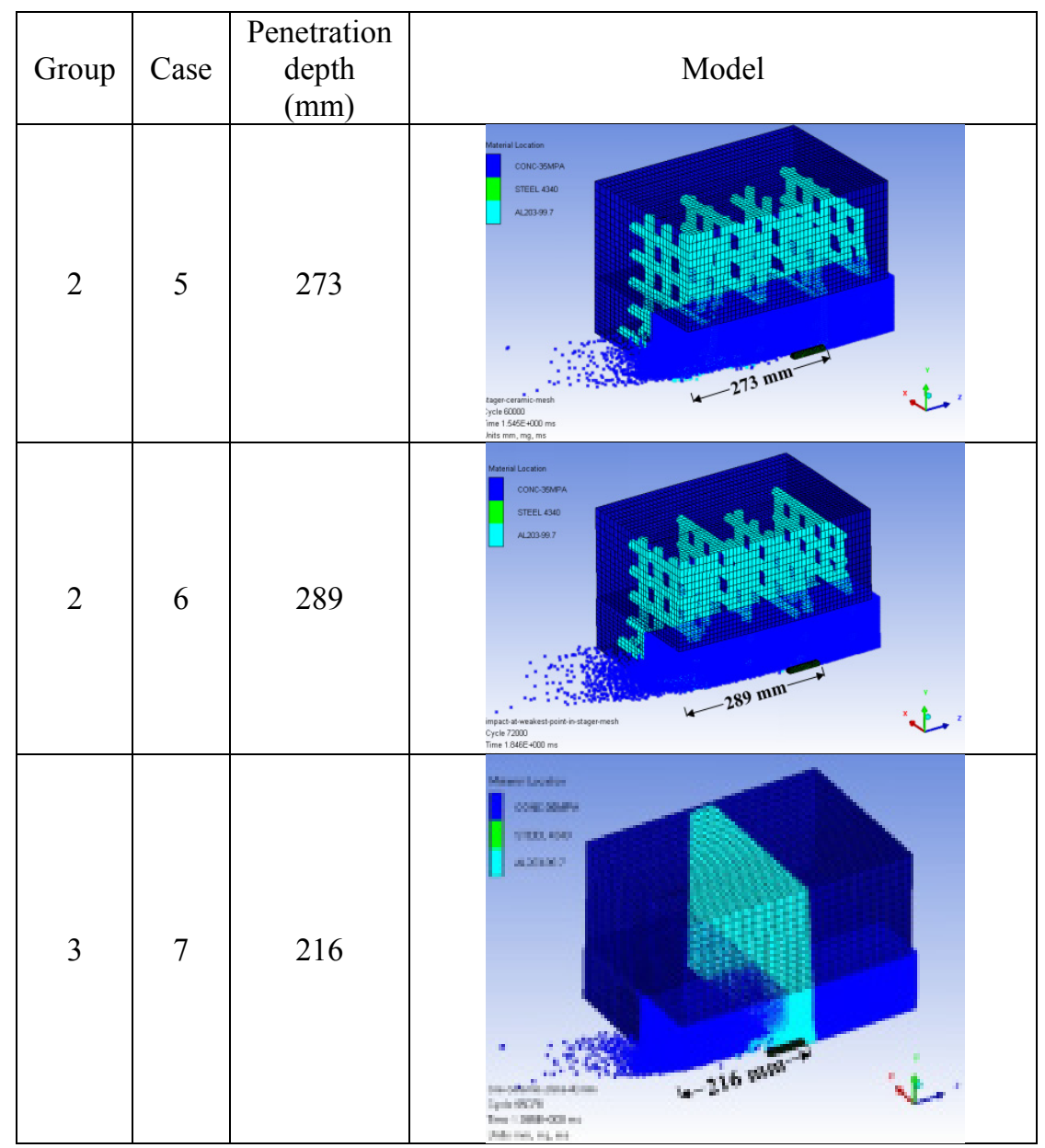

\section{References}

[1] Imran Latif Q.B, Rahman I.A, Ahmad Zaidi A.M., Development of Empirical Formula Prediction on Critical Impact Energy for Perforation Phenomena on Concrete Structures, Journal of Mathematics Research, 3(1), 83-87 (2011).

[2] Rahman I.A., Imran Latif Q.B, Ahmad Zaidi A.M., Norazman M.N., Shohaimi A., Simulation Study on Effect of diameter and CRH Ratio of Ogive Nose Hard Missile on Required Critical Impact Energy for Penetration of Concrete Targets, Journal of International Review on Modeling and Simulations (IREMOS), 4(3):1373-1381 (2011). 
[3] M. E. Mohamed, E.M. Eltehawy, I. M. Kamal, A. A. Aggour, "Experimental Analysis of Reinforced Concrete Panels Penetration Resistance" Proceedings of the $13^{\text {th }}$ International Conference on Aerospace Sciences and Aviation Technology, ASAT-13," May 26-28 (2009).

[4] "AUTODYN Interactive Non-Linear Dynamic Analysis software", Theory Manual, Century Dynamics Company, Inc. (2005).

[5] Olivier F.J. Meuric, Jim Sheridan, Cathy O'Caroll, Richard A. Clegg and Colin J. Hayhurst "Numerical Prediction of Penetration into Reinforced Concrete using a Combined Grid based and Meshless Lagrangian approach." $10^{\text {th }}$ International Symposium on Interaction of the Effects of Munitions with Structures, May 7-11 (2001), California.

[6] Tu Z, Lu Y. Modifications of RHT material model for improved numerical simulation of dynamic response of concrete, International Journal of Impact Engineering (2010), doi:10.1016/j.ijimpeng.2010.04.004 . 